\title{
Predicting an adrenal crisis: can we do it?
}

\author{
Ashley Grossman ${ }^{1}$
}

Received: 2 January 2018 / Accepted: 11 February 2018 / Published online: 1 March 2018

(c) Springer Science+Business Media, LLC, part of Springer Nature 2018

Primary adrenal failure is a condition which should be readily treated with a normal quality of life: we have long been aware of the basic pathophysiology. We are able to essentially fabricate the deficient hormones, and we have sophisticated assays to assess replacement adequacy. Nevertheless, normalisation of the quality of life, avoidance of adrenal crises, and attainment of a normal mortality, appear to be difficult goals to achieve [1]. As clinicians, we weave between the Scylla of under-treatment with consequent fatigue and general ill-health, and the ever-present risk of adrenal crises, as opposed to the Charybdis of overtreatment and the metabolic syndrome. Indeed, even in a highly experienced unit with skilled staff and good patient training, the risk of adrenal crises seems to be ever present [2]. Such patient training includes warnings of 'sick day' rules, emergency injection packs, and in some cases specific patient training days or evenings. It is important to know that subcutaneous injection can be virtually as effective as the standard suggestion of intramuscular hydrocortisone [3], although it should still be emphasised that the advice to 'double the oral dose' during febrile illnesses has hardly any firm evidence base. Nevertheless, in spite of all this activity, adrenal crises and their consequent morbidity and indeed mortality remain stubbornly present concerns in our patients' lives.

In the current issue of Endocrine, Meyer et al. [4] from Frankfurt, Germany, have studied whether a longitudinal assessment of quality of life could in some way help predict (and thus be used to avoid) such adrenal crises. A study of 110 patients over 10 months showed a prevalence of adrenal crises slightly higher than previously published (possibly due at least in part to the cohort including self-referred patients from a patient organisation, and thus possibly

Ashley Grossman

ashley.grossman@ocdem.ox.ac.uk

1 Oxford Centre for Diabetes, Endocrinology and Metabolism, University of Oxford, Oxford, UK especially aware of their adrenal fragility); they found a trend for patients with a varying quality of life to show an increased likelihood to undergo an adrenal crisis, although the study was probably underpowered to demonstrate a suitably significant effect. However, what the authors refer to as 'precrises', requiring an increased dose of hydrocortisone replacement, were associated with a lower quality of life scores. While there is a danger in these findings being tautological, as patients generally less 'well' would be expected to more readily increase their dose of hydrocortisone from time to time, the findings do generally support the view that patients with fluctuating or generally low quality of life are more likely to require increased or emergency doses of hydrocortisone. While the authors do not link this to any specific hydrocortisone dose regimen, these new data do suggest we should be especially vigilant in alerting patients who report fatigue or discomfort with their replacement to any warning signs of impending crisis.

Some 70 years after the first use of corticosteroid replacement we are still some way from giving our adrenally insufficient patients the type of replacement they deserve. There appear to be subtle defects in immune function and inflammatory parameters in patients on conventional replacement which may be lessened by slowrelease preparations [5], while others recommend prednisolone as an alternative glucocorticoid replacement [6]. Whatever the case, we are still some way from knowing how to provide the optimal replacement regimen, and how to avoid life-threatening adrenal crises. All research efforts in this direction should be commended-but we are not there yet.

\section{Compliance with ethical standards}

Conflict of interest The author declares that he has no conflict of interest. 


\section{References}

1. A. Grossman, G. Johannsson, M. Quinkler, P. Zelissen, Perspectives on the management of adrenal insufficiency: Clinical insights from across Europe. Eur. J. Endocrinol. 169, R165-R175 (2013)

2. S. Hahner, C. Spinnler, M. Fassnacht et al., High incidence of adrenal crisis in educated patients with chronic adrenal insufficiency: a prospective study. J. Clin. Endocrinol. Metab. 100, 407-416 (2015)

3. S. Hahner, S. Burger-Stritt, B. Allolio: Subcutaneous hydrocortisone administration for emergency use in adrenal insufficiency. Eur. J. Endocrinol. 169, 147-154 (2013)

4. G. Meyer, M. Koch, E. Herrmann et al., Longitudinal AddiQoL scores may identify higher risk for adrenal crises in Addison's disease. Endocrine, https://doi.org/10.1007/s12020-017-1513-0 (2018) in press

5. A.M. Isidori, M.A. Venneri, C. Graziadio, C. Simeoli et al., Effect of once-daily, modified-release hydrocortisone versus standard glucocorticoid therapy on metabolism and innate immunity in patients with adrenal insufficiency (DREAM): a single-blind, randomised controlled trial. Lancet Diabetes Endocrinol. [Epub ahead of print] (2017)

6. D.J.F. Smith, H. Prabhudev, S. Choudhury, K. Meeran, Prednisolone has the same cardiovascular risk profile as hydrocortisone in glucocorticoid replacement. Endocr. Connect 68, 766-772 (2017) 